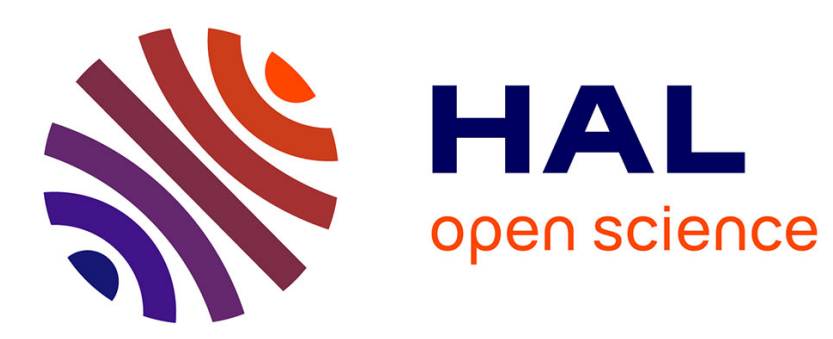

\title{
Possible boundary discontinuities of the tilt angle in nematic liquid crystals
}

\author{
C. Oldano, G. Barbero
}

\section{To cite this version:}

C. Oldano, G. Barbero. Possible boundary discontinuities of the tilt angle in nematic liquid crystals. Journal de Physique Lettres, 1985, 46 (10), pp.451-456. 10.1051/jphyslet:019850046010045100 . jpa00232543

\section{HAL Id: jpa-00232543 https://hal.science/jpa-00232543}

Submitted on 1 Jan 1985

HAL is a multi-disciplinary open access archive for the deposit and dissemination of scientific research documents, whether they are published or not. The documents may come from teaching and research institutions in France or abroad, or from public or private research centers.
L'archive ouverte pluridisciplinaire HAL, est destinée au dépôt et à la diffusion de documents scientifiques de niveau recherche, publiés ou non, émanant des établissements d'enseignement et de recherche français ou étrangers, des laboratoires publics ou privés. 
Classification

Physics Abstracts

$61.30 \mathrm{G}$

\title{
Possible boundary discontinuities of the tilt angle in nematic liquid crystals
}

\author{
C. Oldano (*) and G. Barbero (**) \\ Dipartimento di Fisica, Politecnico Corso Duca degli Abruzzi, 2410129 Torino, Italy \\ and Gruppo Nazionale Struttura della Materia del Consiglio Nazionale delle Ricerche, $\left({ }^{*}\right)$ U.R. di \\ Torino, $\left({ }^{* *}\right)$ U.R. di Cosenza, Italy
}

(Reçu le 3 janvier 1985, révisé le 19 mars, accepté le 27 mars 1985)

\begin{abstract}
Résumé. - On considère l'énergie de surface de Dubois-Violette et Parodi dépendant des premières dérivées de l'orientation du directeur nématique. On montre qu'en l'écrivant comme un simple terme de surface, on ne peut résoudre le problème de trouver les configurations stables du directeur. D'autres informations sont nécessaires sur les interactions donnant lieu à cette énergie. En modélisant simplement cette interaction, on montre que son effet principal est d'imposer une variation non négligeable de l'orientation du directeur près de la surface, quand l'orientation spontanée est inclinée. Toutes les méthodes utilisées précédemment pour trouver les configurations stables du directeur doivent être reconsidérées lorsque les énergies de surface contiennent des termes en gradient de l'orientation du directeur.
\end{abstract}

\begin{abstract}
The boundary energy term, introduced by Dubois-Violette and Parodi, which depends on the first derivatives of the nematic liquid crystal director, is considered. It is shown that if it is written as a purely surface term, the problem of finding stable director configurations cannot be solved. Further information is required about the interaction forces giving rise to this energy term. On the basis of a simple model for such an interaction, it is shown that its main effect is to give a non negligible variation of the tilt angle in a thin boundary layer, when the easy axis is tilted. It is also shown that all the previously used methods for finding the stable director configurations, when surface energy terms dependent on the director derivatives are present, must be reconsidered.
\end{abstract}

The stable equilibrium configurations of a nematic sample are derived from a variational principle, i.e. by requiring that the total free energy $F_{\mathrm{T}}$ be a minimum. In the frame of the continuum theory $F_{\mathrm{T}}$ is obtained by integrating a continuous function $f$ which represents the free energy density. In the bulk this function depends only on the director components $n_{i}$ and on their spatial derivatives $n_{i, j}\left(=\partial n_{i} / \partial x_{j}\right)$, whereas in a boundary layer it also depends explicitly on the distance of the considered point from the limiting wall. Actually, the order parameters and all the related quantities are known to have different values in the bulk and near to the 
limiting walls [1]. When the thickness $r$ of the boundary layer is very small with respect to the sample dimensions, $F_{\mathrm{T}}$ is generally written in the form [2] :

$$
F_{\mathrm{T}}=\iiint_{V} f_{\mathrm{b}}\left(n_{i}, n_{i, j}\right) \mathrm{d} V+\oint_{S} G\left(n_{i}, n_{i, j}\right) \mathrm{d} S,
$$

where $V$ is the sample volume and $S$ its boundary, $f_{\mathrm{b}}$ is the bulk free energy density, whose expression is considered valid in the whole sample, and $G$ is a surface energy term which takes into account the interfacial interactions and the variations of the physical parameters near to the boundary surface. The director configurations thus found are, therefore, only approximations of the actual ones. In this paper it is shown that these approximations are good only in the case where the surface energy term does not depend on the normal derivative of the director components. In the opposite case a standard method for the variational problem does not exist. In the literature two approaches are found, which give different expressions for the director configuration $[3,4]$. It is shown that none of these approximations, which are represented by functions with continuous first order derivatives, are acceptable and a new approach is proposed. More precisely, it is shown that functions having a discontinuity at the boundary surface, may represent a better approximation for the physical problem. However for some forms of the function $G\left(n_{i}, n_{i, j}\right)$ this discontinuity becomes infinite and the problem of minimizing the free energy can only be solved if a further assumption on the free energy dependence through the boundary layer is explicitly made. This is exactly the case when $G$ is assumed to have the form

$$
G=h(\mathbf{n} \cdot \mathbf{u}) \operatorname{div} \mathbf{n}
$$

where $\mathbf{u}$ is a unit vector normal to the surface and $h$ an " elastic constant $»$. A term of this kind was proposed by Dubois-Violette and Parodi on the basis of symmetry considerations [5]. It is also obtained when the bulk free energy term $K_{13} \operatorname{div}(\mathbf{n} \operatorname{div} \mathbf{n}$ ), introduced by Nehring and Saupe $[6,7]$, is integrated over the whole sample.

First we analyse the solutions given in literature [3, 4, 8-13] (point 1). Then we show that : (2) these solutions do not minimize the total free energy; (3) a rigorous analysis is possible only if the range $r$ of the interaction forces, giving rise to the boundary energies, are taken into account; (4) the main effect of the term (2) is to give a strong distortion of the director configuration in the boundary layer.

1. - We consider a nematic film placed between the planes $z=0$ and $z=d$, in the absence of external fields, with strong homeotropic anchoring at the surface $z=0$ and weak anchoring at the surface $z=d$, with easy axis close to the sample normal. Let us first write the total free energy $F$ per unit area in the form

$$
F\{\theta(z)\}=\int_{0}^{d}(k / 2) \theta^{\prime 2} \mathrm{~d} z+(w / 2)\left(\theta_{1}-\Theta_{1}\right)^{2}+h \theta_{1} \theta_{1}^{\prime}
$$

where $\theta=(\mathbf{n u}), \theta_{1}=\theta(d), \theta^{\prime}=\mathrm{d} \theta / \mathrm{d} z$ and $\Theta_{1}$ is the angle made by the easy axis with the surface normal. It can be noticed that in equation (3) the last term corresponds to the surface energy given by equation (2) with the approximation of considering $\sin \theta_{1}=\theta_{1}$. The strong anchoring at the plane $z=0$ gives the boundary condition

$$
\theta(0)=0 \text {. }
$$

The Euler-Lagrange equation of the variational problem is

$$
\theta^{\prime \prime}(z)=0 \text {. }
$$


The solution of equation (5), with the boundary condition (4), is

$$
\theta(z)=\theta_{1}(z / d)
$$

where $\theta_{1}$ must be considered a free parameter, owing to the weak anchoring condition at $z=d$. In reference [4], this parameter is found by inserting the function $\theta(z)$ given by equation (6) into equation (2), and minimizing the function $F\left(\theta_{1}\right)$ thus obtained. This gives

$$
F\left(\theta_{1}\right)=(k / 2 d) \theta_{1}^{2}+(w / 2)\left(\theta_{1}-\Theta_{1}\right)^{2}+(h / d) \theta_{1}^{2} .
$$

$F\left(\theta_{1}\right)$ is a quadratic form having a unique minimum for

$$
\theta_{1}=\Theta_{1}\{w d /(w d+k+2 h)\} .
$$

In references [3, 8-13], a different method is used, which gives a solution of the same kind as that given by (6) with

$$
\theta_{1}=\Theta_{1}\{w d /(w d+k+h)\}
$$

2. - Let us now show that no function which satisfies equation $(5)$ in the closed interval $[0, d]$ can minimize the functional (3).

Let $\theta(z)$ be any function satisfying equation (5) with the boundary condition (4), i.e. a function given by (6), and $\tilde{\theta}(z)=\theta(z)+\varepsilon v(z)$ a new function close to $\theta(z)$, satisfying the same boundary condition. This means that $\varepsilon$ is a small parameter and that $v(0)=0$. The first variation of the total free energy $F$ is easily found to be

$$
\delta F=(\varepsilon / d)\left\{\left[(w d+k+h) \theta_{1}-w d \Theta_{1}\right] v(d)+h \theta_{1} v^{\prime}(d)\right\} .
$$

One may notice that $\left(8^{\prime}\right)$ gives the $\theta_{1}$-value for which the coefficient of $v(d)$ vanishes, whereas (8) gives the value which minimizes $F$ in the class of the solution of the Euler-Lagrange equation, considered valid in the closed interval $[0, d]$.

A simple inspection of equation (9) shows that, starting from any solution $\theta(z)$ of the kind (6), one can further decrease the total free energy by simply changing this function near to the point $z=d$, in order to obtain an increase or a decrease of $v^{\prime}(d)$, according to the sign of the parameter $h$. As a simple example, let us consider a variation of the type

$$
v_{n}(z)=(z / d)\left\{1-(z / d)^{n}\right\} .
$$

Two variate functions $\widetilde{\theta}_{n}\left(z, \theta_{1}, \varepsilon\right)=\theta(z)+\varepsilon v_{n}(z)$ are plotted in figure 1a for $n=10$ and $n=50$ (dashed and dotted lines respectively; the values of the other parameters are given in the caption). Figure $1 \mathrm{~b}$ is a plot of $F\left(n, \theta_{1}, \varepsilon\right) v s$. $\varepsilon$ for the same $n$-values. One may notice that near to $\varepsilon=0$, $F$ monotonically decreases by increasing $\varepsilon$, clearly showing that solutions of the kind (6) do not minimize the total free energy. We further notice that :

- the absolute minimum of $F\left(n, \theta_{1}, \varepsilon\right)$ decreases on increasing $n$, thus suggesting the limit $n \rightarrow \infty$ is to be considered,

- in this limit the variate function $\tilde{\theta}_{n}\left(z, \theta_{1}, \varepsilon\right)$ satisfies the Euler-Lagrange equation (5) in all the open interval $[0, d]$, but not in the $z=d$ point, where a finite discontinuity in $\tilde{\theta}(z)$ and an infinite discontinuity in $\widetilde{\theta}^{\prime}(z)$ appear. More precisely, in the class of the functions $\widetilde{\theta}_{n}\left(z, \varepsilon, \theta_{1}\right)$ the absolute minimum of the functional $F$ is given by the function

$$
\left\{\begin{array}{l}
\theta(z)=\left(\theta_{1}+\Delta \theta\right)(z / d), \text { for } z<d, \\
\theta(d)=\theta_{1},
\end{array}\right.
$$




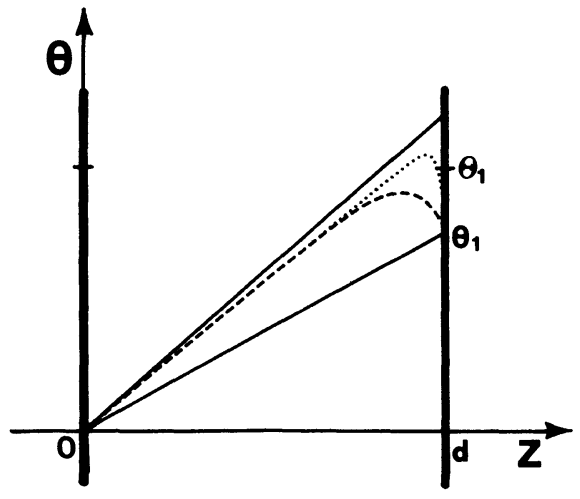

a)

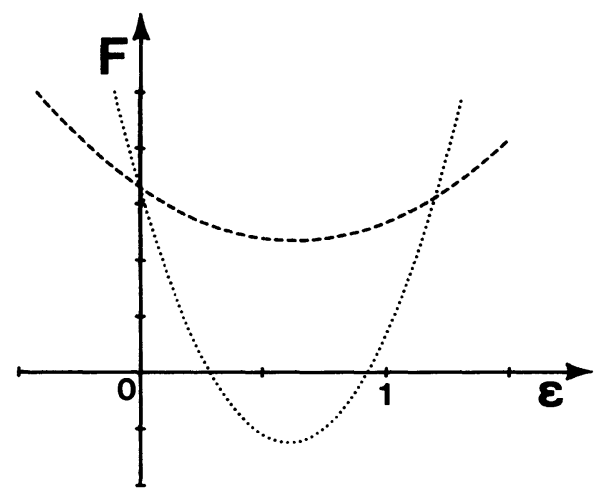

b)

Fig. 1. - Director configurations (a) and free energy $F v s$. $\varepsilon$, (b) for $k: w d: h=2: 10: 0.6$. The dashed and dotted lines in (a) represent $\theta_{n}=\theta(z)+\varepsilon v_{n}(z)$, for $n=10$ and 50 respectively and for $\varepsilon=0.5\left(\theta(z)\right.$ and $v_{n}(z)$ are given by Eqs. (6), (7) and (10)). The corresponding lines in (b) represent the behaviours of the free energy (given by Eq. (3)) vs. $\varepsilon$, in arbitrary units. Similar behaviours, with a minimum for $\varepsilon \neq 0$, are obtained for any $\theta_{1}$-value. The upper and lower full lines of figure la represent the director configurations given by equation (11) and by equations (6) and (8) respectively.

where $\theta_{1}$ is given by equation (8) and $\Delta \theta=2 \theta_{1} h / k$. The functions given by equations (8) and (11) are plotted in figure la (lower and upper full lines respectively). One may notice that if $h$ is not negligible with respect to $k$ the two functions are quite different. As the functional class $\left\{\widetilde{\theta}_{n}\right\}$ is an extension of the functional class defined by equation (6), the function (11) is to be considered as a better approximation of the actual solution than those given in 1. The question if this function is to be considered as the solution of our variational problem, is open. In fact we are interested in founding physically more significative solutions, represented by continuous functions. In the following points 3 and $\mathbf{4}$ it is shown that, in order to find a solution for the variational problem, further assumption about the ranges of the boundary interactions must be made. A possible solution, which is in any case discontinuous, will be found as the limit of the continuous solutions, obtained by taking into account the ranges of the interactions.

Let us first stress some important points :

i) Since $v(d)$ and $v^{\prime}(d)$ can be independently changed, the requirement that $F$ be identically zero gives two boundary conditions, which cannot generally be satisfied by functions depending on a unique parameter $\theta_{1}$. This means that the variational problem has, generally, no solutions in the class of the functions which satisfy the Euler-Lagrange equation in the closed interval [0,d].

ii) The above example suggests that the solution of our variational problem must be searched in the class of the functions with a boundary discontinuity. Obviously this means that the EulerLagrange equation (5) must be considered valid only in the bulk, and not at the boundary point $z=d$.

iii) With a similar procedure it is easy to show that the functionals of the type (1), where a surface energy term which explicitly depends on the director derivatives is present, have, generally, no analytical solutions. More generally such a difficulty arises for the functionals of the type (1), in all cases where the volume density $f_{\mathrm{b}}$ and the surface density $G$ depend on derivatives of the same order. Consequently, for the case of nematic liquid crystals, the surface terms which only depend on $\theta$ and those which also depend also on $\theta^{\prime}$ play very different roles.

3. - As previously noticed, the free energy expressions (1) and (3) must be considered only as an approximation of the actual ones. In fact the surface energy terms arise from interactions 
having non zero ranges. Let us assume that the two surface terms which appear in equation (3) come from different interactions, having ranges $r_{w}$ and $r_{h}$ respectively. The corresponding energies should be written as volume terms which are to be added to the bulk terms, and which are not zero only in boundary layers of thicknesses $r_{w}$ and $r_{h}$. In these layers new terms are therefore to be added to the Euler-Lagrange equation (5), which must only be considered valid in the bulk.

If we assume that the surface energy terms which appear in (3) are obtained from the correct energy expression by some limit operation $r_{w}, r_{h} \rightarrow 0$ the difficulties pointed out in 2 disappear. In fact :

1) In such a limit some type of discontinuity is expected. A discontinuity in the function obviously introduces a further free parameter. Consequently the contradiction pointed out in 2, where a function with only one free parameter must satisfy two boundary conditions, is removed ;

2) The fact that (5) must be considered valid only in the bulk is now clear. In fact in the boundary layer the Euler-Lagrange equation is not given by equation (5). By considering the limit $r_{w}$, $r_{h} \rightarrow 0$ one simply reduces to the boundary point the region where (5) does not hold.

With the help of the above considerations, let us now try to find physically meaningful solution for our problem. We first observe that from the very fact of associating interaction ranges $r_{w}$ and $r_{h}$ to the boundary energy terms, a further very important consequence necessarily follows. In fact the bulk director configuration evidently depends on the ratio $r_{w} / r_{h}$. Consequently in any limit operation the correct ratio must be maintained. The information contained in this ratio is lost when the energy terms are simply written as purely surface terms.

In order to obtain a definite solution, further information on the thickness of the boundary layer and on the free energy density within this layer is needed. Let us now assume that :

a) $r_{w}$ is negligible with respect to $r_{h}$ and strong anchoring conditions are imposed at the surface $z=d$, i.e. :

$$
\theta_{1}=\Theta_{1}
$$

Equation (12) takes into account the effect of the energy term depending on $w$, which can therefore be omitted in the total free energy expression.

b) The free energy density associated to the other boundary term is given by

$$
f=(k / 2) \theta^{\prime 2}+\bar{h}(z) \theta \theta^{\prime},
$$

where $\bar{h}(z)$ is a continuous function which is identically zero for $z<d-r_{h}$ and satisfies the relation

$$
\int_{d-r_{h}}^{d} \bar{h}(z) d z=h .
$$

In a first approximation, we assume that, in the boundary layer, $\theta(z)$ is nearly constant and equal to $\Theta_{1}$. With these hypotheses, the Euler-Lagrange equation is

$$
k \theta^{\prime \prime}(z)+\bar{h}^{\prime}(z) \Theta_{1}=0 .
$$

By considering that for $z<\left(d-r_{\mathrm{h}}\right)$ the quantities $\bar{h}(z)$ and $\bar{h}^{\prime}(z)$ are zero, one easily obtains

$$
\theta^{\prime}(z)=\theta_{\mathbf{B}}^{\prime}-\left(\Theta_{1} / k\right) \bar{h}(z),
$$

where $\theta_{\mathbf{B}}^{\prime}$ is the bulk first derivative of the tilt angle $\theta(z)$.

By equation (16), in the hypothesis $r_{h} \ll d$, the change of $\theta$ through the boundary layer is easily found to be

$$
\Delta \theta=\theta\left(d-r_{h}\right)-\theta(d)=\Theta_{1}(h / k)-\theta_{\mathrm{B}}^{\prime} r_{h} \approx \Theta_{1}(h / k) .
$$


Equation (17) shows that the approximation of considering $\theta$ nearly constant in the boundary layer is good only if $h$ is small with respect to $k$.

4. - Some comments on the solution found and on the underlying hypotheses are needed. We recall that, in addition to the assumptions given by equations (12) and (13), we have assumed : i) $\theta \ll \pi$; ii) strong anchoring at surface; iii) $r_{w} \ll r_{h}$. If the assumption i) is dropped, a similar analysis gives

$$
\Delta \theta \approx(h / 2 k) \sin \left(2 \Theta_{1}\right) .
$$

The main effects of the $h$-dependent energy term is to give a distortion of the director configuration in the boundary layer. The term is therefore associated to strongly distorting forces, which vanishes only for $\theta_{1}=0$ and $\theta_{1}=\pi / 2$ i.e. for homeotropic and planar configurations.

The thickness of the boundary layer depends on the range of the interations, the distortion angle $\Delta \theta$ depends on the ratio $h / k$.

In [7] it is shown that the induced dipole-induced dipole interaction between the molecules of the nematic gives $h$ and $k$ values of comparable magnitude. Obviously the actual value of $h$ depends on all interactions between the nematic-nematic and nematic-substrate molecules. To date no experimental determination of this parameter has been given in the literature (indeed no experimental evidence has been presented for the very existence of such a term). The assumptions ii), iii) and that given by equation (13) have only been made in order to obtain an analytical solution. If assumptions ii) and iii) are dropped, the actual solution strongly depends on the free energy expression in the boundary layer and on the ranges $r_{w}$ and $r_{h}$

A discussion of the validity of the assumption (13) can only be made in framework of a theory relating to the origin of this energy term, which is beyond the aim of this paper. An extension of the Nehring and Saupe theory given in [7], could give rise to new $\theta^{\prime}$-dependent terms in the boundary free energy expression. Here we have used the more simple boundary energy term which in the limit $r_{h} \rightarrow 0$, reduces to the surface energy term (2). In the same limit a boundary discontinuity in $\theta(z)$ appears, given by equations (17) and (18), which are $r_{h}$-independent.

\section{Acknowledgments.}

Acknowledgments are due to G. Durand for fruitful discussions.

\section{References}

[1] See for instance Cognard, J., Mol. Cryst. Liq. Cryst. Suppl. Ser. 1 (1982) 1.

[2] Barbero, G., Madhusudana, N. V., Durand, G., Z. Naturf. A 39 (1984) 1066.

[3] Derzhanski, A. I., Hinov, H. P., Phys. Lett. A 56 (1976) 465.

[4] Barbero, G., Strigazzi, A., J. Physique Lett. 45 (1984) 857.

[5] Dubois-Violette, E., Parodi, O., J. Physique Colloq. 30 (1969) C4-57.

[6] OsEen, C. W., Faraday Soc. 29 (1933) 833.

[7] Nehring, J., Saupe, A., J. Chem. Phys. 54 (1971) 337 and 56 (1972) 5527.

[8] Hinov, H. P., J. Physique Lett. 38 (1977) 215.

[9] Derzhanski, A. I., Hinov, H. P., J. Physique 38 (1977) 1013.

[10] Derzhanski, A. J., Hinov, H. P., Phys. Lett. A 62 (1977) 36.

[11] Hinov, H. P., Derzhanski, A. I., J. Physique Colloq. 40 (1979) C3-305.

[12] Barnik, M. I., Blinov, L. M., Korkishko, T. V., Umanski, B. A. and Chrigrinov, V. G., Mol. Cryst. Liq. Cryst. 99 (1983) 53.

[13] Barnik, M. I., Blinov, L. M., Korkishko, T. V., Umanski, B. A. and Chrigrinov, V. G., Sov. Phys. JETP 58 (1984) 102. 